\title{
Quality of life and its demographic predictors among workers at a plastic factory in Malaysia: a cross-sectional study
}

\author{
Asem Iyad Ahmed Alnabih ${ }^{1}$, Belal Aldabbour ${ }^{2}$, Mohd Faizal Bin Mat Tahir ${ }^{1}$, Nor Kamaliana Khamis ${ }^{1}$ \\ ${ }^{1}$ Mechanical and Manufacturing Engineering Department, Faculty of Engineering \& Built Environment, Universiti Kebangsaan \\ Malaysia, Bangi, Malaysia \\ ${ }^{2}$ Neuroscience Department, Faculty of Medicine, Islamic University of Gaza, Gaza, Palestine
}

\begin{tabular}{l}
\hline Article Info \\
\hline Article history: \\
Received Aug 30, 2021 \\
Revised Nov 9, 2021 \\
Accepted Nov 20, 2021 \\
\hline
\end{tabular}

Keywords:

Demographic predictors

Plastic factory

Quality of life

WHOQOL-BREF

\begin{abstract}
Quality of life (QOL) is an individualized measure that reflects a person's subjective feelings towards the different aspects of his or her life and incorporates them into his overall health evaluation. The WHOQOL-BREF is a QOL measurement tool that has been validated in worldwide and local studies, with good reliability and sensitivity. WHOQOL-BREF questionnaire was used to evaluate the QOL of 89 workers at a plastic factory in Selangor, Malaysia. These were compared using t-test and Spearman's bivariate correlation test to assess for significant correlations and predictors of performance in the different domains. The performance of the sample, both overall and for individual domains, was significantly lower than reported in previous studies. Local workers, highly educated workers, workers with shorter employment, and workers who did not take overtime performed significantly better than their respective counterparts. Also, lower education, foreign nationality, longer employment at the factory, overtime, and crushing jobs were associated with lower QOL scores. Studies evaluating QOL in industrial workers in Malaysia are scarce. Our sample is more diverse than the previous similar studies from Malaysia, and hence it offers new insights into the QOL of plastic industrial workers in the country.
\end{abstract}

This is an open access article under the CC BY-SA license.

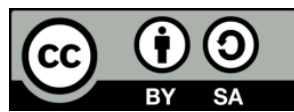

\section{Corresponding Author:}

Mohd Faizal Bin Mat Tahir

Mechanical and Manufacturing Engineering Department, Faculty of Engineering and Built Environment,

Universiti Kebangsaan Malaysia

43600 UKM Bangi, Selangor, Malaysia

Email: mfaizalmt@ukm.edu.my

\section{INTRODUCTION}

Quality of life (QOL) is one of the three main pillars in a concept of understanding and measuring people's well being. Under QOL dimension including the health status, work and life balanced, education and skills, civic engagement and governance, social connections, environmental quality, personal security and subjective wellbeing [1]. The World Health Organization (WHO) defines QOL as "individuals" perceptions of their position in life in the context of the culture and value systems in which they live and in relation to their goals, expectations, standards, and concerns" [2].

Measuring QOL is a complex process owing to the diverse aspects of life that different groups of individuals or researchers may perceive as worthy of assessment. Hence, many measurement instruments have been developed over the years, each instrument having its strengths and limitations. WHOQOL-BREF is an abbreviated version of WHOQOL-100, which contains 26 items divided into four domains, physical (seven questions), psychological (six questions), environmental (eight questions), social relations (three questions), and additionally, two self-assessment questions that assess rated QOL and satisfaction with 
health. WHOQOL-BREF has been established as a cross-cultural instrument for the assessment of QOL, with good to excellent psychometric reliability and validity [3]-[5].

Since the WHOQOL-BREF questionnaire is shorter and easier to administer, it is ideal for the industrial setting where workers may be discouraged from filling the longer version. The effective use of WHOQOL-BREF in the industrial setting has been demonstrated in studies from different countries [6]-[8]. Also, the Malaysian version of the questionnaire has been validated in several studies across different settings [9], and its cultural sensitivity [10], environment impact [11], [12], and clinical with specificity or not were demonstrated [13]-[17].

Previously there a lot of QOL studies in Malaysia but majority were mainly conducted in clinical settings such as either due to drinking behaviour [13], physical disability [14], lifestyle [15] or even health condition [16], [17], and they used various tools for measurement. Moreover, studies evaluating industrial workers in Malaysia are limited. Isahak [6] in his study looked on ergonomic aspect and QOL from the food and textile sector's respondent. However, in his studies the noise exposure criteria are subjectively from respondent perception. Other studies [18] conducted focusing only male worker from automotive assembly in Malaysia to find the relationship psychosocial works factor towards HRQOL. Previous study [19] also noted about the potential workers working under high noise exposure level due to the nature of the industry. Unfortunately, none of the previous local studies assessed about the QOL among all the workers including the foreign workers in Malaysia. Thus, this study aims to measure QOL and its predictors in a plastic factory in Malaysia, both among national and foreign workers. The plastic industry is one of the most vibrant industries in Malaysia's manufacturing sector and was estimated at USD 3,305.39 million, by revenue, in 2017. The industry is projected to expand at an estimated compound annual growth rate (CAGR) of 5.27\% during the forecast period 2018-2023. Having such a big industry that employs thousands of workers indicates that maintaining the welfare and health of the employees in the industry is paramount.

\section{RESEARCH METHOD}

\subsection{Study design and setting}

This cross-sectional study was conducted at a plastic factory in Selangor, Malaysia. The factory has six different production and packaging lines (named A-F) and the crushing department and administration officers. Overall, the facility employs 213 national and foreign workers from both genders, divided over three work shifts. Production lines have different machines, products, and number of workers. Each machine emitted a different noise pressure level thus produce different noise working environment in production line or department. The production lines have the most significant number of workers and constitute the largest factory area when combined with the crushing and packaging departments.

\subsection{Population and case identification}

The study sampled 89 respondents, who were workers from the first shift as it incorporated workers from different nationalities, genders, age groups, and departments (production line workers, crushers, packaging workers, and officers). Eight-nine was the minimal number of workers required for the production lines to be fully functional.

\subsection{Research protocol}

Ethical approval was obtained from the Ethics Committee at the Faculty of Engineering at the National University of Malaysia under code number: JEP-2019-529. Permission for using the translated Malaysian and the English versions of the WHOQOL-BREF questionnaire was obtained on $16^{\text {th }}$ January 2020 through the licensing portal at the WHO website prior to the initiation of the study. Both versions of the questionnaire were subsequently administered to randomly chosen workers in each of the six production lines, packaging department, crushing department, and officers. Verbal consent was obtained from each worker. Then, the questionnaire was explained to each worker, and instructions were given to answer questions based on their feelings over the previous two weeks. Each worker filled the questionnaire individually without assistance.

\subsection{Demographic predictors determination}

The independent variables selected were female gender, having a college degree, non-Malaysian nationality, having over three years of employment at the factory, taking overtime shifts, non-married social status, and working non-crushing jobs. Predictors were selected to determine if there was any relationship between each of the independent variables and QOL. Having a college degree is generally linked to having a higher job position given to the worker. Experts recommend employment duration of three years as the cutoff period for the time needed to learn new skills and build job qualifications. Overtime was defined as working for at least 30 extra minutes after the full eight-hour working day. Some workers worked up to 12 
hours daily. Crushing jobs were distinguished from the other assignments because they involved working in a particularly high-noise environment.

\subsection{Statistical analysis}

The QOL survey results were converted into percentages where a domain was excluded from the count if the worker did not answer more than one question in that domain, as per the instructions of the WHO. The quality of life scale was calculated for each of the four domains, and the scale was categorized as: very poor (0-20), poor (21-40), neither poor nor good (41-60), good (61-80), and very good (81-100).

Independent samples t-test was used to determine significant interactions between the different groups of each independent variable. Then, the independent variables were compared to each domain to determine predictors of performance. Spearman's bivariate correlation test was used to test each of these variables against the individual QOL domains to check for significance and correlation. Subsequently, the different domains were tested for correlation between each other. Finally, the predictors were tested against each question individually to determine which variables predicted a higher score for that question.

Variables selected by each procedure were evaluated using their significant level ( $\rho$-value) to include the preliminary main effect model. In this study, the significance level $\rho$ was set at $\rho<0.05$. The correlation was represented by $r$ where the value was interpreted as weak correlation $(r<0.3)$, fair correlation $(0.3<\mathrm{r}<0.05)$, moderate correlation $(0.5<\mathrm{r}<0.7)$, and good correlation $(0.7<\mathrm{r})$.

\section{RESULTS AND DISCUSSION}

\subsection{Reliability and validity test}

The questionnaire was distributed to a group of experts, postgraduate researchers and three managers on the plastic factory to ensure the questionnaire's clarity. The reliability tests indicated a good acceptance in all QOL domains. Test results (Cronbach's Alpha) were above 0.7 for all domains, as shown in Table 1. This table shows that the survey was reliable and respondent was able to answer correctly and consistent in their answer.

Table 1. The results of the reliability test

\begin{tabular}{ccc}
\hline Reliability test & Cronbach's Alpha & N of items \\
\hline WHOQOL domain 1 & .759 & 7 \\
WHOQOL domain 2 & .742 & 6 \\
WHOQOL domain 3 & .726 & 3 \\
WHOQOL domain 4 & .702 & 7 \\
\hline
\end{tabular}

\subsection{Results}

Figure 1 illustrates the cohort characteristics of the respondents. The mean WHOQOL-BREF score for all workers was 45.28 (range: 14.68-78.13), which can be interpreted as neither poor nor good (41-60) QOL. Table 2 demonstrates the statistical distribution of QOL based on domains (Domain I: physical health, Domain II: psychological health, Domain III: social relationships, and Domain IV: environmental). The lowest scores in all four domains were reported by foreign workers, while the highest scores were reported by the local workers, who also included the highly educated workers. The mean total score was under 50 for each individual domain (neither poor nor good), where the highest score was recorded by Domain II, followed by Domain I, Domain IV, and Domain III. Table 2 demonstrates the significant interactions between categories $(\rho<0.05)$. It was found that gender and marital status were not significant as the $\rho$ value was above 0.05 .

Edimansyah et al. [18] found that $53.7 \%$ of Malaysian workers in the automotive assembly industry have good or very good overall QOL. The performance by domain in that cohort was higher than the cohort in this study, where the mean scores were 67.50 in physical health, 60.63 in phycological health, and 67.50 in the social domain and 60.00 in environmental health. On the other hand, a study by Isahak et al. [6] reported good or very good QOL, where physical health recorded a mean score of $88.13 \%$, phycological health $81.90 \%$, social relative $85.51 \%$, and environmental health $95.37 \%$.

Few factors could have contributed to these differences of QOL obtained in the different studies. The study by Edimansyah et al. [18] included local (Malaysian) male workers only. The physical health of the average Malaysian citizen is better than that of citizens from neighboring countries, which could have impacted the overall QOL reported by participants of this study as shown in Table 3. 


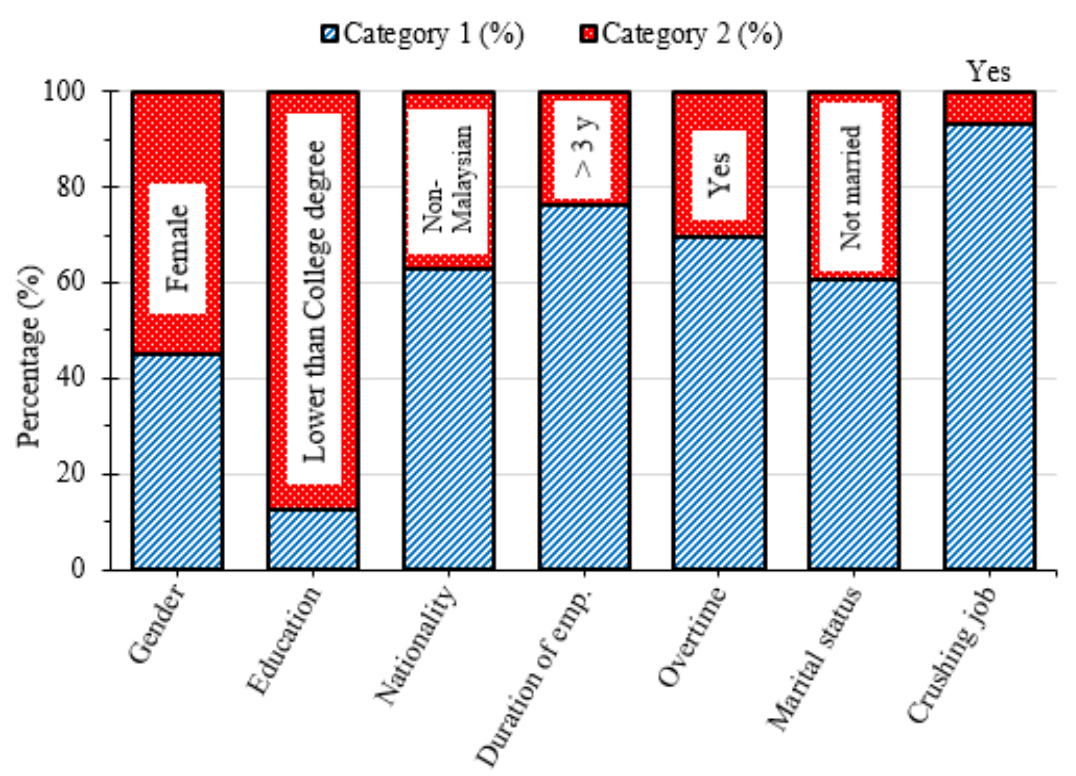

Figure 1. Percentage of respondent's cohort characteristics divided into two categories

Table 2. Statistical distribution of QOL based on four domains

\begin{tabular}{lcccc}
\hline & $\begin{array}{c}\text { Domain I } \\
\text { (physical health) }\end{array}$ & $\begin{array}{c}\text { Domain II } \\
\text { (psychological health) }\end{array}$ & $\begin{array}{c}\text { Domain III } \\
\text { (social relationship) }\end{array}$ & $\begin{array}{c}\text { Domain IV } \\
\text { (environmental) }\end{array}$ \\
\hline Number of workers & 89.00 & 89.00 & 89.00 & 89.00 \\
Mean & 46.83 & 47.61 & 42.84 & 43.86 \\
Median & 46.43 & 50.00 & 41.67 & 43.75 \\
Std. Dev. & 15.66 & 14.68 & 16.10 & 15.00 \\
Variance & 245.08 & 215.39 & 259.23 & 224.93 \\
Range & 75.00 & 75.00 & 75.00 & 78.13 \\
Minimum & 7.14 & 12.50 & 0.00 & 6.25 \\
Maximum & 82.14 & 87.50 & 75.00 & 84.38 \\
\hline
\end{tabular}

Table 3. Mean QOL score for each independent variable category and their interactions

\begin{tabular}{|c|c|c|c|c|c|}
\hline Variables & Groups & Mean overall QOL & Max overall QOL & Min overall QOL & $\rho$ \\
\hline \multirow[t]{2}{*}{ Gender } & Male & 44.70 & 71.50 & 18.01 & \multirow{2}{*}{0.455} \\
\hline & Female & 46.58 & 75.67 & 26.56 & \\
\hline \multirow{2}{*}{ Education } & College degree & 62.14 & 75.67 & 45.16 & \multirow{2}{*}{0.000} \\
\hline & Lower than a college degree & 43.41 & 63.67 & 18.01 & \\
\hline \multirow[t]{2}{*}{ Nationality } & Malaysian & 48.28 & 75.67 & 26.56 & \multirow{2}{*}{0.007} \\
\hline & Non-Malaysian & 41.41 & 63.67 & 18.01 & \\
\hline \multirow[t]{2}{*}{ Duration of employment } & Under three years & 48.70 & 75.67 & 24.80 & \multirow{2}{*}{0.000} \\
\hline & More than three years & 36.13 & 59.15 & 18.01 & \\
\hline \multirow[t]{2}{*}{ Overtime } & No & 48.49 & 75.67 & 26.56 & \multirow{2}{*}{0.001} \\
\hline & Yes & 39.40 & 59.60 & 18.01 & \\
\hline \multirow[t]{2}{*}{ Marital status } & Married & 47.49 & 75.67 & 18.01 & \multirow{2}{*}{0.077} \\
\hline & Not married & 43.01 & 65.65 & 24.80 & \\
\hline \multirow[t]{2}{*}{ Crushing job } & No & 47.04 & 75.67 & 26.56 & \multirow{2}{*}{0.001} \\
\hline & Yes & 32.49 & 44.49 & 18.01 & \\
\hline
\end{tabular}

However, the study by Isahak et al. [6] was conducted on food and textile workers. Both of those industries are considered less demanding compared to heavy industries such as automotive and manufacturing. The study was also restricted to local workers (Malaysian). Additionally, the average weekly overtime was shorter than our study, with an average of 3.34 hours of overtime per week compared to four overtime work hours per day for most of the working days in our cohort, which ultimately impacted the results and contributed to the lower QOL reported in our study.

Other affecting factors that may have contributed to the lower QOL score in our study include the insecurity and uncertainty felt around the time of data collection. This study was conducted in March 2020 where the COVID-19 pandemic was beginning to spread panic worldwide. Here, financial and social uncertainty may have clouded the respondents' judgment. Additionally, the research data and analysis might 
have been predisposed for bias since this study involved fewer respondents than the two other local studies. Of note, all three studies used the same evaluation tool (WHOQOL-BREF) and applied the same modality (self-reporting by workers). Table 4 shows the combination of interactions between the predictors and the domains. As discussed earlier, the significance is accepted if $\rho<0.05$, meanwhile correlation is interpreted as good at $0.7<$ r, moderate if $0.5<r<0.7$, fair if $0.3<r<0.5$, and weak if $r<0.3$.

Table 4. Interactions between predictors and four domains

\begin{tabular}{lcccccccc}
\hline \multicolumn{1}{c}{ Predictor } & \multicolumn{2}{c}{ Domain I } & \multicolumn{2}{c}{ Domain II } & \multicolumn{2}{c}{ Domain III } & \multicolumn{2}{c}{ Domain IV } \\
& (physical health) & \multicolumn{2}{c}{ (psychological health) } & \multicolumn{2}{c}{ (social relationship) } & \multicolumn{2}{c}{ (environment) } \\
& $\rho$ & $\mathrm{R}$ & $\mathrm{P}$ & $\mathrm{r}$ & $\mathrm{P}$ & $\mathrm{r}$ & $\mathrm{\rho}$ & $\mathrm{r}$ \\
\hline Gender & 0.277 & 0.117 & 0.016 & 0.260 & 0.112 & -0.165 & $<0.001$ & 0.386 \\
Education & $<0.001$ & 0.392 & $<0.001$ & 0.492 & 0.515 & 0.070 & $<0.001$ & 0.516 \\
Nationality & 0.011 & -0.267 & $<0.001$ & -0.453 & 0.009 & -0.275 & $<0.001$ & -0.731 \\
Duration of employment & $<0.001$ & -0.489 & 0.001 & -0.347 & 0.961 & -0.005 & 0.043 & -0.215 \\
Overtime & $<0.001$ & -0.397 & $<0.001$ & 0.573 & 0.001 & -0.343 & $<0.001$ & -0.725 \\
Marital status & 0.549 & -0.064 & 0.346 & -0.101 & 0.012 & -0.267 & 0.051 & -0.207 \\
Crushing job & $<0.001$ & -0.430 & $<0.001$ & -0.443 & 0.005 & 0.294 & $<0.001$ & -0.420 \\
\hline
\end{tabular}

Overall, Domain III (social relationship) had no significance towards the predicator designed except for overtime (extra work hours). Although Domain I (physical health) was quite significant towards the predictors, the correlations were either fair or weak. Domain II (physiological health) showed that the overtime predictor was significant and had a moderate correlation $(\rho<0.001, r=0.573)$. Interestingly, Domain IV (environmental) contained one moderate correlation with education $(\rho<0.001, \mathrm{r}=0.516)$, and two high correlations with nationality $(\rho<0.001, \mathrm{r}=-0.731)$ and overtime $(\rho<0.001, \mathrm{r}=-0.725)$.

A significant and positive correlation was demonstrated between overtime and Domain I (physical health), as working extra hours is understandably more exhausting and might cause health problems over the long term. Besides that, having extra work hours might also be associated with unhealthy eating habits, as workers may resort to fast foods instead of home-cooked meals. In addition, education level, nationality, and overtime were found to be significant and had a positive correlation with Domain IV (environmental).

Also, education plays a role in determining one's salary, and therefore it is indirectly associated with the state of their environment. College-level workers had much more secure positions, and the COVID-19 pandemic was less likely to jeopardize their payroll compared to the lower position workers. This further corroborates the findings of previous studies that demonstrated a strong relationship between QOL and education [6], [20], [21]. However, because of COVID-19 pandemic extending over more than a year, the pandemic hit economies and health globally and impacted all the layers across societies [22]-[27]. For nationality, it is understandable that foreign workers (in this case, the non-Malaysian) have additional financial and legal obligations compared to the local worker, which puts them under more environmental pressures. Overtime was also associated with performance in the fourth domain (environmental domain). These correlations signify the impact of variables related to work environment (e.g., noise) on QOL and illustrate how lengthy exposure to these variables may negatively affect QOL, which was shown in this study and in other local and international studies [6], [28].

Regarding gender, duration of employment, marital status, and crushing job, these variables were not significantly correlated with overall QOL. These correlations may be explained by the fact that all $(100 \%)$ workers in the crushing line, where work conditions are more challenging, were all males, while most $(81.90 \%)$ of workers in the packaging line, where work conditions are better, were females. In addition, the workers in the crushing line were rotated with others to comply with the health standards according to the health policy as those workers are exposed to higher risk (e.g., high noise, workload, and others). Thus, working a crushing job had a small effect on the environmental factors.

\subsection{Strength and limitation}

The strengths of this study include the diverse characteristics of sampled workers and the several lines of statistical analysis that were performed. Also, it comes from an area where similar studies are rare, and it reflects QOL of workers in this geographical area during the height of COVID-19 pandemic first wave, just a few days before the first total lockdown in Malaysia. However, there are few limitations to this study. First, self-report questionnaires (one reference source) may predispose to common method biases. Thus, it is recommended that more research be done using different approaches to overcome this issue. Second, the sample size was limited due to the conditions surrounding the data collection period. Future studies are encouraged to sample larger cohorts and assess QOL among workers from different industries, genders, and nationalities in Malaysia.

Int J Public Health Sci, Vol. 11, No. 1, March 2022: 106-112 


\section{CONCLUSION}

The mean overall most of QOL score was identified as medium/average marks neither poor nor good. However, education workers (degree holder) noted a slightly good QOL, and worker who works more than three years, working overtime and working in crushing department (noisy environment) resulting a slight poor on their QOL. Working overtime has a moderate correlation with psychological health and high correlation with environment domain. In terms of contribution to the industry, this study provides a good future reference to industrial managers about indicators that relate to the health and QOL of their workers such as overtime planning that show have a correlation with the domain of QOL.

\section{ACKNOWLEDGEMENTS}

The authors would like to express sincere gratitude to Universiti Kebangsaan Malaysia and the Ministry of Higher Education, Malaysia under Geran Galakan Penyelidik Muda (GGPM-2019-014) for funding part of the project.

\section{REFERENCES}

[1] OECD, How's life?: Measuring well-being, vol. 9789264121. OECD, 2011.

[2] World Health Organization Division of Mental Health, "WHOQOL-BREF : introduction, administration, scoring and generic version of the assessment: field trial version," World Health Organization. 1996, [Online]. Available: https://apps.who.int/iris/handle/10665/63529 (accessed

[3] J. Gagliardi, C. Brettschneider, and H. H. König, "Health-related quality of life of refugees: a systematic review of studies using the WHOQOL-Bref instrument in general and clinical refugee populations in the community setting," Conflict and Health, vol. 15, no. 1, p. 44, Dec. 2021, doi: 10.1186/s13031-021-00378-1.

[4] F. Koohi, S. Nedjat, M. Yaseri, and Z. Cheraghi, "Quality of life among general populations of different countries in the past 10 years, with a focus on human development index: A systematic review and meta-analysis," Iranian Journal of Public Health, vol. 46, no. 1, pp. 12-22, 2017.

[5] Z. Cheraghi, A. Doosti-Irani, S. Nedjat, P. Cheraghi, and S. Nedjat, "Quality of life in elderly Iranian population using the QOLbrief questionnaire: A systematic review," Iranian Journal of Public Health, vol. 45, no. 8, pp. 978-985, 2016.

[6] M. Isahak et al., "The association of workplace exposures on quality of life in small and medium enterprises workers: a crosssectional study in four in ASEAN countries," Asia-Pacific Journal of Public Health, vol. 29, no. 4, pp. 315-327, May 2017, doi: $10.1177 / 1010539517699060$.

[7] S. Y. Yang, D. J. Hsu, C. M. Yen, and J. H. Chang, "Predictive factors of life quality among packaging workers in Taiwan," Health Promotion International, vol. 34, no. 4, pp. 751-759, Aug. 2019, doi: 10.1093/heapro/day029.

[8] J. O. Asante, M. J. Li, J. Liao, Y. X. Huang, and Y. T. Hao, "The relationship between psychosocial risk factors, burnout and quality of life among primary healthcare workers in rural Guangdong province: A cross-sectional study," BMC Health Services Research, vol. 19, no. 1, p. 447, Dec. 2019, doi: 10.1186/s12913-019-4278-8.

[9] Y. B. Cheung, K. K. Yeo, K. J. Chong, E. Y. H. Khoo, and H. L. Wee, "Measurement equivalence of the English, Chinese and Malay versions of the World Health Organization quality of life (WHOQOL-BREF) questionnaires," Health and Quality of Life Outcomes, vol. 17, no. 1, p. 67, Dec. 2019, doi: 10.1186/s12955-019-1130-0.

[10] R. Zakaria and A. A. Razak, "The effectiveness of a cultural-based support group for malay dementia caregivers in Kelantan, Malaysia: a pre-post intervention study," ASEAN Journal of Psychiatry, vol. 18, no. 1, pp. 39-49, 2017.

[11] M. Simis, A. Awang, and K. Arifin, "From ex-landfill to public park: impact on local community's quality of life and living environment," Procedia - Social and Behavioral Sciences, vol. 222, pp. 763-771, Jun. 2016, doi: 10.1016/j.sbspro.2016.05.157.

[12] R. Ismail, M. H. Hafezi, and R. M. Nor, "The effect of demographic characteristic to quality of life and happiness in Malaysia," Research Journal of Applied Sciences, Engineering and Technology, vol. 7, no. 1, pp. 198-205, Jan. 2014, doi: 10.19026/rjaset.7.240.

[13] A. Yee, A. S. A. Adlan, R. R. Rashid, H. Habil, and K. Kamali, "Validation of the alcohol use disorders identification test (AUDIT)-Bahasa Malaysia version among a group of alcohol users," Journal of Substance Use, vol. 20, no. 4, pp. 229-233, Jul. 2015, doi: 10.3109/14659891.2014.894588.

[14] M. M. A. Razak, M. Z. Tauhid, N. F. Yasin, and F. A. Hanapiah, "Quality of life among lower limb amputees in Malaysia," Procedia - Social and Behavioral Sciences, vol. 222, pp. 450-457, Jun. 2016, doi: 10.1016/j.sbspro.2016.05.135.

[15] A. F. Mat Ludin, N. A. Mohamed Nor, S. Omar, S. N. I. Isa, R. Ghoshal, and M. Z. A. Kamaruddin, "Physical activity and health related quality of life among non-academic staff of a university," Jurnal Sains Kesihatan Malaysia, vol. 13, no. 2, pp. 69-75, Nov. 2015, doi: 10.17576/jskm-2015-1302-08.

[16] Z. Harun and S. Ahmad, "Premature ejaculation and quality of life among men atending Klinik Kesihatan Jaya Gading, Kuantan," International Journal of Public Health Research, vol. 8, no. 1, pp. 878-884, 2018.

[17] N. Ibrahim, S. S. L. Teo, N. C. Din, A. H. A. Gafor, and R. Ismail, "The role of personality and social support in health-related quality of life in chronic kidney disease patients," PLoS ONE, vol. 10, no. 7, p. e0129015, Jul. 2015, doi: 10.1371/journal.pone.0129015.

[18] B. A. Edimansyah, B. N. Rusli, L. Naing, B. A. Mohamed Rusli, and T. Winn, "Relationship of psychosocial work factors and health-related quality of life in male automotive assembly workers in Malaysia," Industrial Health, vol. 45, no. 3, pp. 437-448, 2007, doi: 10.2486/indhealth.45.437.

[19] A. I. A. Alnabih, M. F. B. M. Tahir, and N. K. Khamis, "Evaluation of occupational noise exposure in a plastic manufacturing industry: a case study," Jurnal Kejuruteraan, vol. 33, no. 3, pp. 699-707, 2021, doi: 10.17576/jkukm-2021-33(3)-28.

[20] M. Gil-Lacruz, A. I. Gil-Lacruz, and M. L. Gracia-Pérez, "Health-related quality of life in young people: the importance of education," Health and Quality of Life Outcomes, vol. 18, no. 1, p. 187, Dec. 2020, doi: 10.1186/s12955-020-01446-5.

[21] R. ZamZam et al., "Schizophrenia in Malaysian families: A study on factors associated with quality of life of primary family caregivers," International Journal of Mental Health Systems, vol. 5, no. 1, p. 16, 2011, doi: 10.1186/1752-4458-5-16.

[22] D. Nasrullah et al., "Psychological impact among health workers in effort to facing the COVID-19 in indonesia," International 
Journal of Public Health Science (IJPHS), vol. 10, no. 1, pp. 181-188, Mar. 2021, doi: 10.11591/ijphs.v10i1.20524.

[23] F. Pulvirenti et al., "Health-related quality of life in common variable immunodeficiency italian patients switched to remote assistance during the COVID-19 pandemic," Journal of Allergy and Clinical Immunology: In Practice, vol. 8, no. 6, pp. 1894-1899.e2, Jun. 2020, doi: 10.1016/j.jaip.2020.04.003.

[24] K. Riiser, S. Helseth, K. Haraldstad, A. Torbjørnsen, and K. R. Richardsen, “Adolescents' health literacy, health protective measures, and health-related quality of life during the COVID-19 pandemic," PLOS ONE, vol. 15, no. 8 august, p. e0238161, Aug. 2020, doi: 10.1371/journal.pone.0238161.

[25] S. Galea, R. M. Merchant, and N. Lurie, "The mental health consequences of COVID-19 and physical distancing: the need for prevention and early intervention," JAMA Internal Medicine, vol. 180, no. 6, pp. 817-818, Jun. 2020, doi: 10.1001/jamainternmed.2020.1562.

[26] P. Pribadi, L. Lolita, R. C. A. Pangestuti, H. Lutfiyati, S. Mareti, and A. Ikhsanudin, "Knowledge and quality of life among Indonesian students during the COVID-19 pandemic," International Journal of Public Health Science (IJPHS), vol. 10, no. 2, pp. 451-458, Jun. 2021, doi: 10.11591/ijphs.v10i2.20819.

[27] A. A. Azlan, M. R. Hamzah, T. J. Sern, S. H. Ayub, and E. Mohamad, "Public knowledge, attitudes and practices towards COVID-19: A cross-sectional study in Malaysia," PLoS ONE, vol. 15, no. 5, p. e0233668, May 2020, doi: 10.1371/journal.pone.0233668.

[28] M. Virtanen, S. A. Stansfeld, R. Fuhrer, J. E. Ferrie, and M. Kivimäki, "Overtime work as a predictor of major depressive episode: a 5-year follow-up of the whitehall II study," PLoS ONE, vol. 7, no. 1, p. e30719, Jan. 2012, doi: 10.1371/journal.pone.0030719.

\section{BIOGRAPHIES OF AUTHORS}
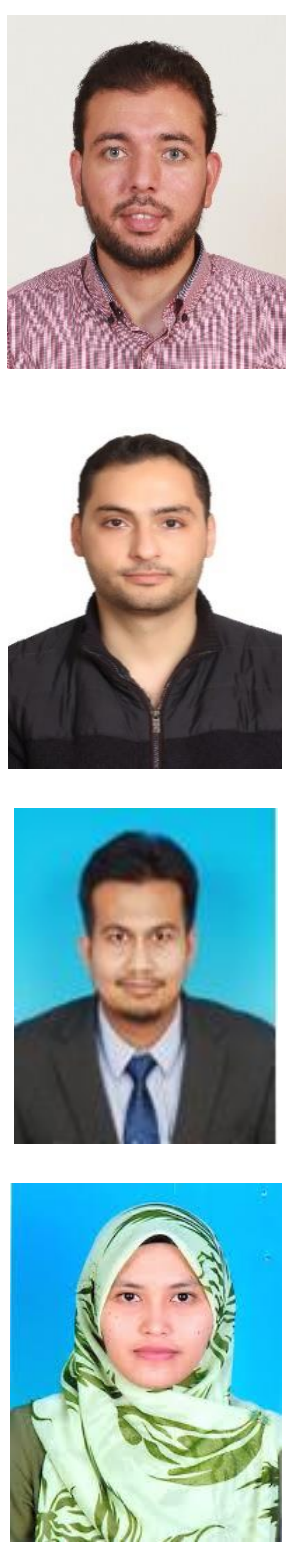

Asem Iyad Ahmed Alnabih (D) SC SC P is an engineer and researcher, is postgraduate student at the national university of Malaysia, Mr. Asem received a Bachelor's degree in Industrial Engineering from the Islamic University of Gaza (IUG), and Master of Science degree in mechanical engineering in the Universiti Kebangsaan Malaysia. His research focuses on the noise, acoustics, health risks, and Ocupational health. For years, He worked at the Gaza Municipality, where he reported directly to the mayor's office. He was put in charge of the Department of Communication and Public Awareness, which was responsible for oversight and management of field research related to different Municipality projects in Gaza. He can be contacted at email: aalnabih@gmail.com.

Belal Aldabbour (D) SC SC P is currently an Assistant Professor of Neuroscience and Neurology at the Faculty of Medicine, Islamic University of Gaza, Gaza, Palestine. Dr. Aldabbour got his MD degree from the Islamic University of Gaza, and then he joined residency training in neurology at King Abdullah University Hospital in Jordan. He is a certified neurologist. He is also interested in research and epidemiology and has several scientific publications in his portfolio. He can be contacted at email: belal90md@gmail.com.

Mohd Faizal Mat Tahir (D) SC S P is an academic at Department of Mechanical and Manufacturing Engineering, Faculty of Engineering and Built Environment, Universiti Kebangsaan Malaysia. He earned both his Bachelor degree and Master degree from Universiti Kebangsaan Malaysia (UKM), in the field of Mechanical Engineering. He obtained his PhD degree at Loughborough University, UK, in 2019 from the school of Aeronautical, Automotive, Chemical and Material Engineering. His research interests are in the field of noise and vibration, such as environment acoustics, material for acoustics and structure borne. He also interests in environment health and safety, ergonomic and digital image correlation research. He can be contacted at email: mfaizalmt@ukm.edu.my.

Nor Kamaliana Khamis (D) SC P P is a Senior Lecturer in Faculty of Engineering and Built Environment, Universiti Kebangsaan Malaysia. She received a Bachelor's degree in Manufacturing Engineering from Universiti Kebangsaan Malaysia, a Master's degree from Universiti Putra Malaysia, and a Doctoral's degree from Universiti Kebangsaan Malaysia and University of Duisburg-Essen, Germany. Her research focuses on the ergonomics, occupational environmental interaction and road safety. She can be contacted at email: kamaliana@ukm.edu.my. 